\title{
The Marsden Morbidity Index: The Derivation and Validation of a Simple Risk Index Scoring System Using Cardiopulmonary Exercise Testing Variables to Predict Morbidity in High Risk Patients Having Major Cancer Surgery.
}

Zeenat Nawoor-Quinn ( $\nabla$ zeenatnawoorquinn@gmail.com )

Royal Marsden Hospital NHS Trust: Royal Marsden NHS Foundation Trust https://orcid.org/00000001-7731-0312

\section{Alex Oliver}

Royal Marsden Hospital NHS Trust: Royal Marsden NHS Foundation Trust

\section{Ravi Raobaikady}

Royal Marsden Hospital NHS Trust: Royal Marsden NHS Foundation Trust

Kabhir Mohammad

Royal Marsden Hospital Chelsea

\section{Stephen Cone}

Royal Marsden Hospital Chelsea

\section{Ramanathan Kasivisvanathan}

Royal Marsden Hospital NHS Trust: Royal Marsden NHS Foundation Trust

\section{Research}

Keywords: Cardiopulmonary Exercise Testing, CPET, Morbidity Score, Major Cancer Surgery, Risk Index Score, Risk Prediction Tool, High Risk Patient, Anaerobic Threshold, Ventilatory Equivalent, Surgery, Exercise, Preoperative Evaluation

Posted Date: July 7th, 2021

DOI: https://doi.org/10.21203/rs.3.rs-668246/v1

License: (1) (i) This work is licensed under a Creative Commons Attribution 4.0 International License. Read Full License 


\section{Abstract}

\section{Background}

Morbidity and mortality risk prediction tools are increasingly being used as part of preoperative assessment of patients presenting for major abdominal surgery. Cardiopulmonary exercise testing (CPET) can predict which patients undergoing major abdominal surgery are at risk of complications. The primary aim of this study was to identify pre-operative variables including those derived from CPET, that were associated with inpatient morbidity in high risk patients following major abdominal cancer surgery. Secondary aims were to use these variables to derive and validate a morbidity risk prediction tool.

\section{Methods}

We conducted a retrospective cohort analysis of consecutive adult patients who had CPET as part of their pre-operative work-up for major abdominal cancer surgery. Morbidity was a composite outcome, defined by the Clavien-Dindo score and/or the Postoperative Morbidity Survey (POMS) score which was assessed on postoperative day 7. A risk prediction tool was devised using variables from the first analysis which was then applied prospectively to a matched cohort of patients.

\section{Results}

A total of 1398 patients were included in the first phase of the analysis between June 2010 and May 2017. Of these, 540 patients (38.6\%) experienced postoperative morbidity. CPET variables deemed significant $(p<0.01)$ were Anaerobic Threshold (AT), Maximal Oxygen Consumption at Maximal Exercise Capacity (VO2 Max) and Ventilatory Equivalent for Carbon Dioxide at Anaerobic Threshold (AT VE/VCO2). In addition to the CPET findings and the type of surgery the patient underwent, eight premorbid variables that were associated with post-operative morbidity were identified. These include age, WHO category, body mass index (BMI), prior transient ischaemic attack (TIA) or stroke, chronic renal impairment, diabetes mellitus, chronic obstructive pulmonary disease (COPD) and cancer stage. Both sets of variables were then combined to produce a validated morbidity risk prediction scoring tool called the Marsden Morbidity Index. In the second phase of the analysis, this tool was applied prospectively to 424 patients between June 2017 and December 2018. With an area under the curve (AUC) of 0.79, this new model had a sensitivity of $74.2 \%$, specificity of $78.1 \%$, a positive predictive value (PPV) of $79.7 \%$ and a negative predictive value of (NPV) of $79 \%$.

\section{Conclusion}

Our study showed that of the CPET variables, AT, VO2 Max and AT VE/VCO2 were shown to be associated with postoperative surgical morbidity following major abdominal oncological surgery. When combined with a number of preoperative co-morbidities commonly associated with increased risk of postoperative morbidity, we created a useful institutional scoring system for predicting which patients will 
experience adverse events. However, this system needs further validation in other centres performing oncological surgery.

\section{Introduction}

As the population becomes more elderly, the incidence of cancer increases concomitantly with other comorbidities. ${ }^{1,2}$ Surgical resection forms the mainstay of treatment for most solid organ tumours. ${ }^{3}$

As a result, major cancer surgery is expected to account for a significant and disproportionate proportion of all healthcare spending in the developed world in the next 20 years. ${ }^{4}$ The ability to objectively predict morbidity preoperatively allows for better targeted resource allocation and optimisation; risk stratification and supports informed decision-making and consent. ${ }^{5}$

In modern day practice, in-hospital mortality following major cancer surgery is low with post-operative morbidity and complications much higher, ${ }^{6,7}$ although surgical postoperative morbidity varies further according to surgical sub-speciality. ${ }^{8}$ Adverse events following major abdominal cancer surgery are often linked to the severity of pre-existing co-morbidities and the functional ability of patients to meet the extra metabolic demands required when undertaking such significant surgery. ${ }^{9,10}$ Cardiopulmonary exercise testing (CPET) is one method considered to be the gold standard, to objectively measure a patient's cardiopulmonary function. ${ }^{11}$ Poor performance on preoperative CPET has consistently been shown to be associated with morbidity following major abdominal cancer surgery. ${ }^{12-15}$ In the UK, CPET is increasingly used for risk prediction as part of a comprehensive preoperative assessment, especially in "high risk" patients prior to major surgery. ${ }^{16,17}$ This study was primarily designed to investigate whether CPET, when combined with other commonly recorded pre-operative variables, were associated with post-operative morbidity in a large mixed cohort of high-risk patients scheduled for major abdominal cancer surgery. The secondary aim was to devise a simple risk prediction model using these significant variables and then prospectively validate this model for our institution.

\section{Methods}

\section{Primary Outcome}

The primary outcome was to identify which preoperative variables, including those derived from CPET, were independently associated with morbidity following major abdominal cancer surgery. This was studied as a retrospective cohort analysis of consecutive adult patients undergoing CPET as part of their pre-operative work-up for major abdominal cancer surgery at a single high volume cancer centre, The Royal Marsden National Health Service Foundation Trust, London SW3 6JJ, between June 2010 to May 2017.

Inclusion criteria were all adult patients $>18$ years of age who had a CPET assessment as part of their pre-operative work up for planned elective major abdominal cancer surgery. Ethical approval was 
obtained from the local institutional review board of the Royal Marsden NHS Foundation Trust and approved as a service evaluation (Reference SE443). The criteria for CPET in patients scheduled for major oncological abdominal surgery at the Royal Marsden NHS Foundation Trust is as follows:

- Patients $>18$ years.

And 1 or more of the following:

- $\leq 4$ metabolic equivalents or equivalent.

- Significant cardiorespiratory comorbidities.

- Exposure to chemotherapy agents which are known to cause cardiotoxicity.

- Do not meet any of the contraindications to CPET as stated by our institutional policy which is in line with the national guidance. ${ }^{16}$

\section{Perioperative Pathway}

All "high risk" patients studied had a pre-operative assessment (fitness of assessment work ups) prior to abdominal cancer surgery. They all had a CPET to assess their functional capacity, which was used to assess fitness for surgery and decision making. Post operatively all patients were admitted to an intensive care or high dependency unit. Pre-operative data was extracted from the electronic patient record (EPR) by the institution's information team who were not involved in the study. Pre-operative comorbidities were documented in the patient's EPR by a nurse and/or doctor at the pre-assessment and were collected based on the ICD-10 (International Statistical Classification of Diseases and Related Health Problems $)^{18}$ definitions. CPET data was extracted from a contemporaneously held database by exercise physiologists who ran the tests.

\section{Secondary Outcome}

The secondary aim was to create a simple risk score that assigns a point score to significant risk factors and then assign the different scores as a risk of morbidity using data from June 2010 - May 2017. This risk scoring system was then validated using prospectively collected data from an 18-month period between June 2017 -December 2018.

\section{Morbidity}

Morbidity was evaluated as categorical data, using validated morbidity scoring system in the ClavienDindo (CD) Complication Grading system ${ }^{19}$ and the Post-operative Morbidity Survey (POMS). ${ }^{20}$

Morbidity was a composite outcome defined as

- Clavien-Dindo score of $\geq 3$ and/or

- Postoperative morbidity survey score of $>1$ at postoperative day (POD) 7 
A CD score $\geq$ grade 3 and/or POMS > 1 on POD 7 during the patient's inpatient hospital stay were both classed as clinically significant complication. ${ }^{21,22}$ Morbidity outcomes were assessed through analysis of the patient's EPR and were recorded routinely by the Royal Marsden's Information Team. If patients included in the study were discharged prior to POD 7, it was assumed that they had no morbidity.

\section{Cardiopulmonary Exercise Testing}

Cardiopulmonary exercise testing was performed and reported by an accredited exercise physiologist. Testing was conducted using the standardised approach recommended by the American Thoracic Society (ATS) and American College of Physicians (ACCP) ${ }^{23}$ in conjunction with the PeriOperative Exercise Testing and Training Society (POETTS) guidelines. ${ }^{16}$ Exercise testing was conducted on an electromagnetically braked cycle ergometer (Ergoselect 200; Ultima CardiO2®; Medical Graphics Corp., St Paul, MN, USA) following resting spirometry. Ventilation and gas exchange were measured using a metabolic cart (Ultima ${ }^{\text {TM }}$ CardiO2 ${ }^{\circledR}$ gas exchange analysis system, MGC Diagnostics, Minnesota, USA). Routine physiological measurements of function included: Work rate (Watts); Spirometric parameters Minute ventilation (VE) and tidal volume (VT); Metabolic gas exchange measurements - 02 consumption (VO2), $\mathrm{CO} 2$ production (VCO2), respiratory exchange ratio ( $\mathrm{RER}=\mathrm{VCO} 2 / \mathrm{VO} 2)$; Ventilatory equivalents for 02 (VE/VO2) and CO2 (VE/VCO2); Cardiovascular variables - heart rate, electrocardiogram (ECG), NIBP and respiratory variables - respiratory rate and oxygen saturation. The CPET data were analysed using Cardioperfect 1.6.2.1105 [Welch Allyn (UK) Ltd, Aston Abbotts, UK] and MedGraphics BreezeSuite 8.5.0.57SP3 (Medical Graphics Corp.)

\section{Analysis methods}

The primary dataset was used to describe the patient characteristics in the morbidity and no-morbidity groups using counts and percentages for the categorical variables and mean/median and standard deviation or interquartile range. Binary logistic regression analysis method was then used in the univariate and multivariate settings to identify the morbidity risk factors. All variables were candidate in the multivariate model analysis. Backward stepwise method was used with a cut-off point ( $p$-value < 0.01 ) for a variable to be included in the fitted multivariate model. Predicted probabilities were obtained for the primary and temporal validation datasets using the post estimation commands of the analysis software, which were then categorized at a cut-off point of 0.5 and summarized into binary classification table (observed and predicted morbidity) for a sensitivity analysis. Sensitivity, specificity, negative and positive predictive values and classification accuracy of the model where calculated in the primary and validation datasets. Similarly, ROC (Receiver Operator Characteristic) were fitted and AUC (Area Under Curve) values obtained. Nomograph was utilized to assign scores to the significant variables in the fitted multivariate logistic regression model to produce graph that can use clinically to identify the patient with high probability of morbidity risk. The preoperative status of the patients is to be scored against the nomograph variables to obtain the total scores which can then be converted to probability to determine their morbidity risk level. STATA version 13 was used for analysis. ${ }^{24}$

\section{Results}


Figure 1 shows the flow through of patients in the study. A total of 8,482 patients were scheduled for major abdominal cancer surgery during the study period, of whom 2,013 $(23.7 \%)$ were deemed high risk. Of these, 615 patients were excluded from analysis either because CPET data were incomplete or they did not proceed to the intended major abdominal surgery following CPET. Reasons for not proceeding with surgery post CPET include patient declining surgery, death before the planned operation date, patient deemed unfit for surgery following a multidisciplinary team decision process and in the event where the surgery was "open-and-close" due to unresectable disease. In total, 1398 patients (704 men and 694 women) underwent CPET followed by the intended abdominal surgery and their data were included in the analysis.

Patient demographics and perioperative characteristics are summarised in Table 1. It also provides a summary of the CPET data measured for all 1398 patients included in the analysis. These were patients who had CPET and abdominal cancer surgery. The median age of these patients was 68 years (range: 21-89). 
Table 1

Preoperative description of patients submitted to cardiopulmonary exercise testing (CPET) prior to major abdominal surgery and summary of CPET outcomes. SD, Standard Deviation; IQR, Interquartile Range

\begin{tabular}{|c|c|}
\hline Variable & Overall Patients $(n=1,398)$ \\
\hline & Median (range) \\
\hline Age (years) & 68 (21-89), (65-78) \\
\hline \multicolumn{2}{|l|}{ Median (range), (IQR) } \\
\hline \multicolumn{2}{|l|}{ Body Mass Index (BMI) } \\
\hline Mean (SD) & $27.98(5.87)$ \\
\hline Median (range), (IQR) & $28.0(12.3-48.4),(24.3-32.0)$ \\
\hline \multicolumn{2}{|c|}{ Anaerobic Threshold (AT) } \\
\hline Mean (SD) & $10.79(2.01)$ \\
\hline Median (range), (IQR) & $10.4(5.5-19.9),(10.0-12.6)$ \\
\hline \multicolumn{2}{|l|}{ V02 Max } \\
\hline Mean (SD) & $17.11(5.74)$ \\
\hline Median (range), (IQR) & $15.8(4.5-37.6),(13.9-18.9)$ \\
\hline \multicolumn{2}{|l|}{ AT VE/VCO2 } \\
\hline Mean (SD) & $35.68(4.39)$ \\
\hline Median (range), (IQR) & $36(20-48),(32-38)$ \\
\hline \multicolumn{2}{|l|}{ Oxygen pulse } \\
\hline Mean (SD) & $9.63(3.00)$ \\
\hline \multirow[t]{2}{*}{ Median (range), (IQR) } & $9(2-14),(9-13)$ \\
\hline & $\mathrm{n}(\%)$ \\
\hline \multicolumn{2}{|l|}{ Age group } \\
\hline$\leq 50$ years & $164(12)$ \\
\hline $51-70$ years & $722(52)$ \\
\hline$>70$ years & $512(37)$ \\
\hline \multicolumn{2}{|l|}{ Gender: } \\
\hline Female & $694(50)$ \\
\hline Male & $704(50)$ \\
\hline
\end{tabular}




\begin{tabular}{|c|c|}
\hline Variable & Overall Patients $(n=1,398)$ \\
\hline \multicolumn{2}{|c|}{ BMI Standard Categories } \\
\hline$<18.5$ & $203(14)$ \\
\hline $18.5-24.99$ & $199(14)$ \\
\hline$\geq 25.0$ & $996(71)$ \\
\hline \multicolumn{2}{|l|}{ Surgery type: } \\
\hline Robotic/Laparoscopic & $356(25)$ \\
\hline Open & $1,042(75)$ \\
\hline \multicolumn{2}{|c|}{$\begin{array}{l}\text { American Society of Anaesthesiologists (ASA) } \\
\text { score: }\end{array}$} \\
\hline ASA 1 & $22(2)$ \\
\hline ASA 2 & $1,076(77)$ \\
\hline \multicolumn{2}{|l|}{ ASA 3} \\
\hline \multicolumn{2}{|c|}{ World Health Organisation (WHO) Category: } \\
\hline 0 & $735(53)$ \\
\hline 1 & $565(40)$ \\
\hline 2 & $53(4)$ \\
\hline 3 & $43(3)$ \\
\hline 4 & $2(0.1)$ \\
\hline \multicolumn{2}{|l|}{ Arrythmia } \\
\hline No & $1,246(89)$ \\
\hline Yes & $152(11)$ \\
\hline \multicolumn{2}{|l|}{ Documented Cirrhosis: } \\
\hline No & $1,307(93)$ \\
\hline Yes & $91(7)$ \\
\hline \multicolumn{2}{|c|}{ Congestive cardiac failure: } \\
\hline No & $1,358(97)$ \\
\hline Yes & $40(3)$ \\
\hline
\end{tabular}




\begin{tabular}{|c|c|}
\hline Variable & Overall Patients $(n=1,398)$ \\
\hline \multicolumn{2}{|l|}{ Diabetic status: } \\
\hline Negative & $1,034(74)$ \\
\hline Non-insulin & $306(22)$ \\
\hline Insulin dependent & $58(4)$ \\
\hline \multicolumn{2}{|c|}{ Chronic Obstructive Pulmonary Disease (COPD): } \\
\hline No & $862(62)$ \\
\hline Yes & $536(38)$ \\
\hline \multicolumn{2}{|l|}{ Previous cardiac event: } \\
\hline No & $975(70)$ \\
\hline Yes & $423(30)$ \\
\hline \multicolumn{2}{|c|}{ Prior transient ischaemic attack (TIA) or stroke: } \\
\hline No & $1,269(91)$ \\
\hline Yes & $129(9)$ \\
\hline \multicolumn{2}{|l|}{ Chronic renal impairment: } \\
\hline No & $1,147(82)$ \\
\hline Yes & $251(18)$ \\
\hline \multicolumn{2}{|l|}{ Number of procedures: } \\
\hline 1 & $782(56)$ \\
\hline 2 & $154(11)$ \\
\hline$\geq 3$ & $462(33)$ \\
\hline \multicolumn{2}{|c|}{ Cancer stage (TNM Classification): } \\
\hline Primary cancer & $1,234(88)$ \\
\hline Local nodal metastases & $140(10)$ \\
\hline Distant metastases & $23(2)$ \\
\hline
\end{tabular}




\begin{tabular}{|l|l|}
\hline Variable & Overall Patients $(\mathbf{n = 1 , 3 9 8 )}$ \\
\hline Surgical categories: & $228(16)$ \\
Hepatobiliary & $340(24)$ \\
Urology & $330(24)$ \\
General surgery & $118(8)$ \\
Colorectal/lower Gastrointestinal (GI) & $234(17)$ \\
Upper Gastrointestinal (GI) & $138(10)$ \\
Gynaecological & $9(0.6)$ \\
Sarcoma &
\end{tabular}

Five hundred and forty patients (36.8\%) experienced morbidity, a composite outcome defined as ClavienDindo score of $\geq 3$ and/or POMS-defined morbidity on POD 7. The CPET variables on univariate analysis associated with postoperative morbidity were AT, VO2 Max, AT VE/VCO2 and Oxygen Pulse (Table 2.1).

Binary logistic regression analysis method was used in the univariate and multivariate settings to identify the morbidity risk factors. Backward stepwise method using $(p<0.01)$ was used to fit the multivariate model. Table 2.1 is the description of the morbidity and no-morbidity patients and summary of the univariate logistic regression results for all 1398 patients included in the primary analysis while Table 2.2 is the summary of the output of the multivariate logistic regression results. 
Table 2.1

Univariate Logistic Regression Analysis. OR, Odds Ratio. 95\% Cl, 95\% Confidence Interval

\begin{tabular}{|c|c|c|c|c|}
\hline Variable & $\begin{array}{l}\text { Morbidity } \\
n=540\end{array}$ & $\begin{array}{l}\text { No morbidity } \\
\mathrm{n}=858\end{array}$ & OR (95\% Cl) & p-value \\
\hline & Median (range) & Median (range) & & \\
\hline Age (years) & & & $1.01(1.00-1.02)$ & 0.013 \\
\hline Mean (SD) & $68.36(9.63)$ & $66.79(12.38)$ & & \\
\hline Median (IQR) & $68(65-78)$ & $68(65-78)$ & & \\
\hline BMI & & & $0.99(0.97-1.01)$ & 0.347 \\
\hline Mean (SD) & $27.79(8.02)$ & $28.10(3.97)$ & & \\
\hline Median (IQR) & $29(18-35)$ & $28(26-32)$ & & \\
\hline AT & & & $0.54(0.50-0.59)$ & $<0.001$ \\
\hline Mean (SD) & $9.62(2.00)$ & $11.53(1.64)$ & & \\
\hline Median (IQR) & $9.6(7.8-11.8)$ & $11.1(10.4-12.6)$ & & \\
\hline V02 Max & & & $0.71(0.69-0.74)$ & $<0.001$ \\
\hline Mean (SD) & $13.20(4.35)$ & $19.57(5.11)$ & & \\
\hline Median (IQR) & $12.9(9.9-14.6)$ & $18.9(15.8-23.4)$ & & \\
\hline AT VE/VCO2 & & & $1.32(1.28-1.37)$ & $<0.001$ \\
\hline Mean (SD) & $38.19(3.70)$ & $34.09(4.03)$ & & \\
\hline Median (IQR) & $36(32-38)$ & $35(30-37)$ & & \\
\hline Oxygen pulse & & & $0.97(0.93-1.00)$ & 0.080 \\
\hline Mean (SD) & $9.46(2.80)$ & $9.75(3.11)$ & & \\
\hline Median (IQR) & $8.9(6.7-13.2)$ & $10.2(6.6-13.2)$ & & \\
\hline Possum Score & & & $0.99(0.97-1.00)$ & 0.023 \\
\hline Mean (SD) & $33.32(6.10)$ & $34.41(9.97)$ & & \\
\hline \multirow[t]{2}{*}{ Median (IQR) } & $34(32-34)$ & $34(28-35)$ & & \\
\hline & n (\%) & $\mathrm{n}(\%)$ & & \\
\hline
\end{tabular}




\begin{tabular}{|c|c|c|c|c|}
\hline Variable & $\begin{array}{l}\text { Morbidity } \\
n=540\end{array}$ & $\begin{array}{l}\text { No morbidity } \\
\mathrm{n}=858\end{array}$ & OR $(95 \% \mathrm{Cl})$ & p-value \\
\hline \multicolumn{5}{|l|}{ Age group } \\
\hline$\leq 50$ years & $46(28)$ & $118(72)$ & 1 & 0.014 \\
\hline $51-70$ years & $289(40)$ & $433(60)$ & $1.71(1.18-2.48)$ & 0.005 \\
\hline$>70$ years & $205(40)$ & $307(60)$ & $1.71(1.17-2.51)$ & 0.006 \\
\hline Gender: & $267(38)$ & $427(62)$ & 1 & 0.907 \\
\hline Female & $273(39)$ & $431(61)$ & $1.01(0.82-1.26)$ & \\
\hline \multicolumn{5}{|l|}{ Male } \\
\hline \multicolumn{5}{|l|}{ BMI Standard Categories } \\
\hline$<18.5$ & $171(84)$ & $32(16)$ & $29.00(16.91-49.59)$ & $<0.001$ \\
\hline $18.5-24.99$ & $31(16)$ & $168(84)$ & 1 & $<0.001$ \\
\hline$\geq 25.0$ & $338(34)$ & $658(66)$ & $2.78(1.86-4.17)$ & $<0.001$ \\
\hline Surgery type: & & & & $<0.001$ \\
\hline Robotic/ Laparoscopic & $28(8)$ & $328(92)$ & 1 & \\
\hline Open & $512(49)$ & $530(51)$ & $11.32(7.55-16.96)$ & \\
\hline \multicolumn{5}{|l|}{ ASA score: } \\
\hline ASA 1 & $9(41)$ & $13(59)$ & 1 & $<0.001$ \\
\hline ASA 2 & $475(44)$ & $601(56)$ & $1.14(0.48-2.69)$ & 0.762 \\
\hline ASA $3 \& 4$ & $56(19)$ & $244(81)$ & $0.33(0.14-0.81)$ & 0.016 \\
\hline WHO category: & $210(29)$ & $525(71)$ & 1 & $<0.001$ \\
\hline 0 & $330(50)$ & $333(50)$ & $2.48(1.99-3.09)$ & \\
\hline \multicolumn{5}{|l|}{$\geq 1$} \\
\hline \multicolumn{5}{|l|}{ Arrythmia } \\
\hline No & $439(35)$ & $807(65)$ & 1 & $<0.001$ \\
\hline Yes & $101(66)$ & $51(34)$ & $3.64(2.55-5.20)$ & \\
\hline \multicolumn{5}{|l|}{ Documented Cirrhosis: } \\
\hline No & $496(38)$ & $811(62)$ & 1 & 0.050 \\
\hline Yes & $44(48)$ & $47(52)$ & $1.53(1.00-2.34)$ & \\
\hline
\end{tabular}




\begin{tabular}{|c|c|c|c|c|}
\hline Variable & $\begin{array}{l}\text { Morbidity } \\
n=540\end{array}$ & $\begin{array}{l}\text { No morbidity } \\
\mathrm{n}=\mathbf{8 5 8}\end{array}$ & $\mathrm{OR}(95 \% \mathrm{Cl})$ & p-value \\
\hline Congestive cardiac failure: & & & & $<0.001$ \\
\hline No & $506(37)$ & $852(63)$ & 1 & \\
\hline Yes & $34(85)$ & $6(15)$ & $9.54(3.98-22.88)$ & \\
\hline \multicolumn{5}{|l|}{ Diabetic status: } \\
\hline Negative & $400(39)$ & $634(61)$ & 1 & 0.300 \\
\hline Non-insulin & $123(40)$ & $183(60)$ & $1.07(0.82-1.38)$ & 0.634 \\
\hline Insulin dependent & $17(29)$ & $41(71)$ & $0.66(0.37-1.17)$ & 0.155 \\
\hline COPD: & & & & $<0.001$ \\
\hline No & $247(29)$ & $619(71)$ & 1 & \\
\hline Yes & $293(55)$ & $243(45)$ & $3.02(2.40-3.76)$ & \\
\hline Previous cardiac event: & & & & $<0.001$ \\
\hline No & $302(31)$ & $673(69)$ & 1 & \\
\hline Yes & $238(56)$ & $185(44)$ & $2.87(2.27-3.63)$ & \\
\hline Prior TIA/stroke: & & & & $<0.001$ \\
\hline No & $446(35)$ & $823(65)$ & 1 & \\
\hline Yes & $94(73)$ & $35(27)$ & $4.96(3.31-7.43)$ & \\
\hline Chronic renal impairment: & & & & $<0.001$ \\
\hline No & $323(28)$ & $824(72)$ & 1 & \\
\hline Yes & $217(86)$ & $34(14)$ & $16.28(11.09-23.90)$ & \\
\hline \multicolumn{5}{|l|}{ Number of procedures: } \\
\hline 1 & $252(32)$ & $530(68)$ & 1 & $<0.001$ \\
\hline$\geq 2$ & $288(47)$ & $328(53)$ & $1.85(1.48-2.30)$ & \\
\hline \multicolumn{5}{|l|}{ Cancer stage: } \\
\hline Primary & $474(38)$ & $760(62)$ & 1 & $<0.001$ \\
\hline Local nodal metastases & $45(32)$ & $95(68)$ & $0.76(0.52-1.10)$ & 0.148 \\
\hline Distant metastases & $20(87)$ & $3(13)$ & $10.69(3.16-36.17)$ & $<0.001$ \\
\hline
\end{tabular}




\begin{tabular}{|llll|}
\hline Variable & $\begin{array}{l}\text { Morbidity } \\
\mathbf{n = 5 4 0}\end{array}$ & $\begin{array}{l}\text { No morbidity } \\
\mathbf{n = 8 5 8}\end{array}$ & OR (95\% Cl) \\
\hline Surgical categories & & & \\
Hepatobiliary & $85(37)$ & $143(63)$ & \\
Urology & $141(41)$ & $199(59)$ & \\
General surgery & $129(39)$ & $201(61)$ & \\
Colorectal/lower GI & $30(25)$ & $88(75)$ & \\
Upper GI & $99(42)$ & $135(58)$ & \\
Gynaecological & $52(38)$ & $86(62)$ & \\
Sarcoma & $3(33)$ & $6(67)$ & \\
\hline
\end{tabular}


Table 2.2

Multivariate model output from backward stepwise selection model including only variables that are significant at $(p<0.01)$ in the multivariate model.

\begin{tabular}{|c|c|c|}
\hline Variable & OR (95\% Cl) & p-value \\
\hline VO2 Max (continuous) & $0.82(0.77-0.86)$ & $<0.001$ \\
\hline AT (continuous) & $0.66(0.61-0.71)$ & $<0.001$ \\
\hline $\begin{array}{l}\text { AT VE/VCO2 } \\
\text { (continuous) }\end{array}$ & $1.33(1.26-1.40)$ & $<0.001$ \\
\hline \multicolumn{3}{|l|}{ COPD: } \\
\hline No & 1 & 0.001 \\
\hline Yes & $1.99(1.33-2.98)$ & \\
\hline $\begin{array}{l}\text { Chronic renal } \\
\text { impairment: } \\
\text { No } \\
\text { Yes }\end{array}$ & $\begin{array}{l}1 \\
7.50(4.29-13.10)\end{array}$ & $<0.001$ \\
\hline \multicolumn{3}{|l|}{ Age group } \\
\hline$>70$ years & 1 & 0.001 \\
\hline $51-70$ years & $0.35(0.18-0.69)$ & 0.003 \\
\hline$<50$ years & $0.25(0.12-0.51)$ & $<0.001$ \\
\hline \multicolumn{3}{|l|}{ Diabetic status: } \\
\hline Negative & 1 & 0.002 \\
\hline Insulin & $2.54(1.52-4.23)$ & $<0.001$ \\
\hline Non-Insulin dependent & $1.02(0.98-1.06)$ & 0.831 \\
\hline \multicolumn{3}{|l|}{ BMI Categories: } \\
\hline$<18.5$ & $6.98(2.91-16.74)$ & $<0.001$ \\
\hline $18.5-24.99$ & 1 & $<0.001$ \\
\hline$\geq 25.0$ & $1.72(0.90-3.30)$ & 0.102 \\
\hline \multicolumn{3}{|l|}{ Surgery type: } \\
\hline Robotic/ Laparoscopic & 1 & $<0.001$ \\
\hline Open & $\begin{array}{l}10.80(5.77- \\
20.19)\end{array}$ & \\
\hline
\end{tabular}




\begin{tabular}{|lll|}
\hline Variable & OR $(\mathbf{9 5 \%} \mathrm{Cl})$ & p-value \\
\hline Cancer stage: & 1 & 0.006 \\
Primary & $1.61(0.84-3.09)$ & 0.153 \\
Local nodal metastases & $11.22(2.31-$ & 0.003 \\
Distant metastases & $54.66)$ & \\
WHO category: & & \\
0 & 1 & $<0.001$ \\
$\geq 1$ & $3.94(2.58-6.03)$ & \\
Prior TIA/stroke: & & 0.002 \\
No & 1 & \\
Yes & $3.07(1.50-6.31)$ & \\
\hline
\end{tabular}

\section{Fitted model classification ability:}

Table 3.1

Fitted model binary classification table (observed and predicted morbidity)

\begin{tabular}{|llll|}
\hline Probabilities & Morbidity n(\%) & No-morbidity n(\%) & Total \\
\hline$\geq 0.5$ (morbidity) & $461(86)$ & $60(7)$ & 522 \\
\hline$<0.5$ (no morbidity) & $77(14)$ & $798(93)$ & 875 \\
\hline Total & 539 & 858 & 1,397 \\
\hline Model prediction strength (Pseudo R2) & 0.59 & \\
\hline
\end{tabular}

$\mathrm{AUC}=\mathbf{0 . 8 1}$

Sensitivity $=75.7 \%$

Specificity $=73.0 \%$

Positive Predictive Value (PV) $=78.5 \%$

Negative PV $=71.2 \%$

Correctly classified $=80.2 \%$

\section{Temporal validation: using the validation dataset}

Description of patients in the validation dataset: Four hundred and twenty-four patients who had surgery in the year June 2017 - December 2018 were used for the model (temporal) validation. The median age 
of these patients was 68 years (range: $49-79$ and IQR: 68-69).

Table 3.2

Temporal dataset binary classification table (observed and predicted morbidity)

\begin{tabular}{|llll|}
\hline Probabilities & Morbidity $\mathbf{n ( \% )}$ & No-morbidity $\mathbf{n}(\%)$ & Total \\
\hline$\geq 0.5$ (morbidity) & $192(84)$ & $43(22)$ & 235 \\
\hline$<0.5$ (no morbidity) & $36(16)$ & $153(78)$ & 189 \\
\hline Total & 228 & 196 & 424 \\
\hline
\end{tabular}

Model Classification Ability:

$\mathrm{AUC}=0.79$

Sensitivity $=74.2$

Specificity $=78.1$

Positive PV $=79.7$

Negative PV $=79.0$

Correctly classified $=71.4$

Appendix 1: Nomograph Variables Predicted Scores 


\begin{tabular}{|c|c|c|c|}
\hline NOMOGRAPH Variables & Predicted Scores & NOMOGRAPH Variables & Predicted Scores \\
\hline COPD & & Chronic Renal Impairment & \\
\hline No & 0.0 & No & 0.0 \\
\hline Yes & 0.5 & Yes & 1.5 \\
\hline CPET: VO2 Max & & BMI categories & \\
\hline 37.60 & 0.0 & Normal & 0.0 \\
\hline 26.57 & 1.6 & Underweight & 1.4 \\
\hline 15.53 & 3.3 & Overweight & 0.4 \\
\hline \multirow[t]{2}{*}{4.50} & 4.9 & & \\
\hline & & Age groups & \\
\hline CPET: AT VE/VCO2 & & $<51$ years & 1.0 \\
\hline 38.67 & 4.2 & $51-70$ years & 0.3 \\
\hline 41.67 & 6.1 & $>70$ years & 0.0 \\
\hline 43.87 & 8.1 & & \\
\hline \multirow[t]{2}{*}{$>45.00$} & 10.0 & Type of Surgery & \\
\hline & & Robotic /Laparoscopy & 0.0 \\
\hline WHO Point Score & & Open & 1.7 \\
\hline $\mathrm{WHO}=0$ & 0.0 & & \\
\hline \multirow[t]{2}{*}{ WHO $>0$} & 1.0 & Diabetes & \\
\hline & & Negative & 0.0 \\
\hline Prior TIA Stroke & & Non-Insulin & 0.1 \\
\hline No & 0.0 & Insulin dependent & 0.8 \\
\hline \multirow[t]{2}{*}{ Yes } & 0.8 & & \\
\hline & & CPET: AT & \\
\hline Cancer stage & & $>13.2$ & 0 \\
\hline Primary & 0.0 & 10.2 & 2.6 \\
\hline Nodal metastases & 0.3 & 8.1 & 5.2 \\
\hline Distant metastases & 1.8 & $<8.0$ & 8.0 \\
\hline
\end{tabular}




\section{Conclusion}

Our study showed that of the CPET variables, AT, VO2 Max and AT VE/VCO2 were shown to be associated with postoperative surgical morbidity following major abdominal oncological surgery. When combined with a number of preoperative co-morbidities commonly associated with increased risk of postoperative morbidity, we created a useful institutional scoring system for predicting which patients will experience adverse events. However, this system needs further validation in other centres performing oncological surgery.

\section{Discussion}

In this retrospective cohort of high-risk patients presenting for major abdominal cancer surgery we found that postoperative morbidity was significantly associated with twelve variables: age, BMI, WHO status, cancer stage (TNM classified), CPET-generated data (AT, VO2 Max and AT VE/VCO2), pre-existing comorbidities (chronic renal impairment, COPD, diabetes mellitus and a previous history of TIA or stroke) and whether a minimally invasive or an open approach was undertaken. These variables were shown to have good strength in discriminating postoperative morbidity in a prospective group of major abdominal cancer surgical patients. Using a scoring system based on the significance of each of these variables on post-operative morbidity, a simple risk scoring system called the "Marsden Morbidity Index" was devised. This index can be used in our institution to predict morbidity in patients scheduled for major abdominal surgery as a means of aiding decision making, consent and resource allocation.

The CPET variables we found to be associated with morbidity were in keeping with findings from previous studies where CPET was evaluated as a risk prediction tool in major abdominal surgery. ${ }^{25-27}$ Our study demonstrated that AT and VO2 Max were significant $(\mathrm{p}<0.001)$ variables at the multivariate analysis level and predictive of poor surgical outcomes. In the perioperative context both have been shown to be strong ergometric predictors of postoperative complications and mortality in a number of cohorts analysing outcomes post major abdominal and thoracic surgery. ${ }^{28-30}$ West et al. ${ }^{31}$ conducted a prospective blinded observational study to investigate for any association between CPET findings and postoperative morbidity after major colonic surgery. Patients who suffered postoperative complications had significantly lower oxygen uptake at lactate threshold, lower VO2 at peak, and higher AT VE/VCO2. These variables were found to be independently predictive of morbidity post rectal cancer surgery and major colonic surgery. Lee et al. ${ }^{32}$ demonstrated a significant association between pre-operative oxygen consumption on a 6-minute walk test and postoperative medical complications $(P<0.01)$ but not surgical and all complications post elective colorectal resection.

In our analysis, VE/VCO2 at anaerobic threshold had the strongest weighting in the model for postoperative morbidity in major abdominal surgery. This is a measure of ventilatory efficiency and is elevated in conditions such as heart failure, pulmonary embolism and chronic lung disease. ${ }^{33}$ It is thus unsurprising that this variable is so strongly associated with morbidity. According to Junejo et al., ${ }^{34}$ CPET findings for preoperative risk assessment before pancreatoduodenectomy showed VE/VCO2 at AT to be 
the only CPET variable independently associated with postoperative morbidity, with an AUC of 0.65 ( $95 \%$ $\mathrm{Cl}$ 0.53-0.77). Similar to our study, CPET was applied in patients deemed high risk and POMS scores were used to assess postoperative morbidity. An AT VE/VCO2 of $\geq 34.5 \mathrm{ml} / \mathrm{kg} / \mathrm{min}$ was found to have a specificity of $84 \%$ and a sensitivity of $47 \%$, with a PPV of $76 \%$ and an NPV of $60 \%$, for POMS-defined morbidity.

Anaerobic threshold $\left(\mathrm{VO}_{2}\right.$ at $\left.\mathrm{AT}\right)$ was a significant $\mathrm{CPET}$ variable associated with postoperative morbidity in this analysis of high-risk patients undergoing major abdominal cancer surgery. This is consistent with one of our previous studies that demonstrated VO2 at AT $<10.2 \mathrm{ml} / \mathrm{kg} / \mathrm{min}$ as a significant predictor of POMS-defined morbidity on POD 3 in patients undergoing major hepatic resection. ${ }^{35}$ Peak VO2 or AT is a measure of aerobic capacity and classified as an important predictor of perioperative morbidity in multiple studies. ${ }^{36-37}$

Objective risk identification and stratification is pivotal in linking preoperative co-morbidities to riskadapted intraoperative approaches and targeted postoperative care pathways. There are multiple grading and risk stratification tools currently in use for surgical patients. Perhaps the most commonly used grading system by anaesthetists is the American Society of Anaesthesiologists (ASA), a system dating back to $1963 .{ }^{38}$ However, this system is largely subjective and does not take into account any surgeryrelated factors. The methodology for risk evaluation has evolved significantly over the years to models that can generate pre-calculated quantitative estimations of morbidity and mortality risks. ${ }^{39}$ These developments also reflect the necessity for objective validated risk assessment tools with advancing perioperative care in order to reduce subjective bias.

The "Marsden Morbidity Index" was developed on the strong advocacy for CPET as an objective risk prediction tool based on current evidence and literature. ${ }^{40}$ However, CPET alone was deemed not enough due to its limitations. ${ }^{41}$ Our aim was to combine CPET variables with premorbid variables to increase acuity in risk prediction. Our study thence demonstrated that the incorporation of CPET variables into a risk prediction tool that also takes other significant clinical variables into account, yields data that can improve the precision of perioperative risks evaluation. The comorbidities we identified are strongly validated in other risk scoring systems ${ }^{42-45}$ currently in use, reflecting the precision of this new model. For this model the AUC to discriminate morbidity was 0.81 and 0.79 in the fitted model binary classification and the temporal validation model respectively.

Premorbid variables deemed significant in the generation of the "Marsden Morbidity Index" risk prediction tool can be further sub-grouped into baseline parameters (age, BMI, WHO category and TNM-classified cancer stage) and chronic conditions (COPD, diabetes mellitus, chronic renal impairment and a previous history of TIA or stroke). The majority of these variables have been strongly validated in multiple risk prediction scores, like $\mathrm{CHA}_{2} \mathrm{DS}_{2}$-VASc, ${ }^{42}$ p-POSSUM, ${ }^{43}$ Lee's Revised Cardiac Risk Index ${ }^{44}$ and SORT ${ }^{45}$ where one or more of these premorbid variables are incorporated in a multifactorial risk-score calculation tool. 
An interesting finding of our analysis showed that a low BMI scored higher than a high BMI. The effect of $\mathrm{BMI}$ on postoperative complications have been long studied with weight taken as a reflection of general health status from a broader perspective. From a preoperative evaluation, it reflects preoperative nutritional status, functional status, presence of co-morbidities, anticipation of intraoperative challenges and a tailored multi-disciplinary team input required for preoptimization and rehabilitation. While obesity is generally assumed to be a risk factor for postoperative adverse events, there is no convincing data to support this assumption. ${ }^{46} \mathrm{~A}$ study published by Tjeertes et al. ${ }^{46}$ to seek more understanding of the obesity paradox revealed that while obesity alone is a significant risk factor for wound infection, more surgical blood loss and a longer operation time, being obese is also associated with improved long-term survival. Complication and mortality rates were found to be significantly worse for underweight patients, who were most at risk of major postoperative complications, including long-term mortality. We also know from current literature that many of the CPET variables, like peak or $\mathrm{VO}_{2} \mathrm{Max}$, are highly correlated with muscle mass. ${ }^{47-48}$ While there is no available data on the direct comparison between CPET outcomes for high versus low BMI in cancer patients, the findings are a cause of concern that patients with low BMI are likely to perform equivocally if not worse than obese patients, which needs confirmation by a more comprehensive investigation.

In addition, our study featured patients who underwent an open laparotomy were more likely to suffer from postoperative complications $(P<0.001)$ when compared to minimally invasive surgery, i.e. roboticassisted or laparoscopy. These findings are in keeping with the literature where the unique benefits and superiority of minimally invasive procedures over open procedures in selected patients have been shown. ${ }^{49} \mathrm{~A}$ systematic review and meta-analysis by Wang et al. comparing the two approaches for pancreatico-duodenectomy showed significant reductions in estimated blood loss, postoperative haemorrhage, transfusion rate, wound infection and length of hospital stay. ${ }^{50}$ Similar findings from comparison between laparoscopy and laparotomy for rectal cancer include reductions in postoperative pain, length of stay, incisional hernia, adhesive bowel obstruction, wound complications, and mortality. ${ }^{51}$ The use of robotic-assisted surgery in the management of cancer continues to increase with numerous evidence in the literature of a shorter convalescence period postoperatively. ${ }^{52}$

In conclusion, we found the CPET variables of AT, VO2 Max and AT VE/VCO2, and a number of preoperative baseline demographics and co-morbidities commonly associated with increased risk of postoperative morbidity, were shown to be associated with postoperative surgical morbidity following major abdominal oncological surgery. Our study shows that the incorporation of CPET variables into a risk prediction tool produces a model with a strong ability to discriminate postoperative complications when morbidity was assessed using a combination of the Clavien-Dindo classification scoring system and the Postoperative Morbidity Survey. Such acuity has the potential to systematically optimize outcomes for surgical intervention.

While this model has helped us create a useful institutional tool for perioperative risks, it needs further validation in other centres performing oncological surgery. To our knowledge, the Marsden Morbidity

Page 21/30 
Index is unique in that is one of only a few validated risk scoring tools that directly incorporates CPET variables as part of their algorithms to predict perioperative outcomes.

\section{Strengths And Limitations}

The strength of this study is the large number of "high-risk" cancer patients that were studied $(n=1398)$. This makes it one of the largest published datasets looking at the association of CPET on post-operative surgical morbidity. This was a strongly validated study and the result reflect the high risk cohort of patients that present to the Royal Marsden Hospital as a tertiary oncological centre.

One of the major limitations of our study is that only high-risk patients based on our institutional criteria had CPET. The ideal study design would be that all patients had CPET to limit bias in the population studied. The risk calculator is thus only valid on our high risk patient cohort. Nonetheless when looking at real world use of CPET, most published data is from a high risk cohort of patients extracted from a general surgical population. Another major limitation was that the study did not account for individual surgical specialities, patient pathways and the fact that the study occurred over a 10 year period where perioperative practices changed. Despite this the variables initially derived as being associated with morbidity were strongly validated in predicting and discriminating (AUC 0.79) in the prospectively studied population. This suggests that despite a number of important factors not being accounted for in the preoperative variables, the model is a strong tool for our population. We would be interested in implementing its use in our institution which may provide further validation of the data.

\section{Declarations}

\section{Availability of data and materials}

The datasets generated during and/or analysed during the current study are available in the Royal Marsden Hospital and the datasets during and/or analysed during the current study are available from the corresponding author on reasonable request.

\section{Affiliations:}

The Royal Marsden Biomedical Research Centre (BRC) and The Institute of Cancer Research, London (ICR)

\section{Acknowledgements}

The authors thank The Royal Marsden's academic partner, The Institute of Cancer Research, London (ICR), which is designated as the UK's National Institute for Health Research (NIHR) Biomedical Research Centre (BRC) dedicated solely to cancer.

\section{Ethics Declaration}


Ethical approval was obtained from the local institutional review board of the Royal Marsden NHS Foundation Trust and approved as a service evaluation (Reference SE443).

\section{Consent for publication}

Not applicable.

\section{Authors Contributions}

ZNQ: reviewed data that were analysed for the purpose of this study between 2010 and 2018, prepared and revised the manuscript. AO: conducted CPET testing and drafted manuscript. RR: conducted CPET testing and drafted manuscript. SC: Chief Clinical Information Officer, collected data and CPET database maintenance. KM: Senior Statistician, ICR; stat advisor, stats analysis and methodologist. RK: Conceived idea of study; results analysis; draft of manuscript. All authors have read and approved the final manuscript.

\section{Corresponding author}

Correspondence to Zeenat Nawoor-Quinn

\section{Funding}

None.

\section{Competing interests}

The authors declare that they have no competing interests.

\section{References}

1. Pedersen JK, Engholm G, Skytthe A, Christensen K. Academy of Geriatric Cancer Research (AgeCare). Cancer and aging: Epidemiology and methodological challenges. Acta Oncol. 2016;55(Suppl 1):712. doi:10.3109/0284186X.2015.1114670. Suppl 1 ) .

2. Atella V, Piano Mortari A, Kopinska J, et al. Trends in age-related disease burden and healthcare utilization. Aging Cell. 2019;18(1):e12861. doi:10.1111/acel.12861.

3. Bakos $\mathrm{O}$, Lawson C, Rouleau S, et al. Combining surgery and immunotherapy: turning an immunosuppressive effect into a therapeutic opportunity. Journal for ImmunoTherapy of Cancer 2018;6:86. doi: 10.1186/s40425-018-0398-7

4. Sullivan R, Alatise OI, Anderson BO, et al. Global cancer surgery: delivering safe, affordable, and timely cancer surgery. Lancet Oncol. 2015;16(11):1193-224. doi:10.1016/S1470-2045(15)00223-5.

5. Moonesinghe SR, Mythen MG, Das P, Rowan KM, Grocott MP. Risk stratification tools for predicting morbidity and mortality in adult patients undergoing major surgery: qualitative systematic review. Anesthesiology. 2013;119(4):959-81. doi:10.1097/ALN.0b013e3182a4e94d. 
6. Weiser TG, Gawande A. Excess Surgical Mortality: Strategies for Improving Quality of Care. In: Debas HT, Donkor P, Gawande A, Jamison DT, Kruk ME, Mock CN, eds. Essential Surgery: Disease Control Priorities, Third Edition (Volume 1). Washington (DC): The International Bank for Reconstruction and Development / The World Bank; April 2, 2015.

7. Morris EJ, Taylor EF, Thomas JD, et al. Thirty-day postoperative mortality after colorectal cancer surgery in England. Gut. 2011;60(6):806-13. doi:10.1136/gut.2010.232181.

8. Endo I, Kumamoto T, Matsuyama R. Postoperative complications and mortality: Are they unavoidable? Ann Gastroenterol Surg. 2017;1(3):160-3. doi:10.1002/ags3.12045. Published 2017 Oct 13.

9. Lee L, Schwartzman K, Carli F, et al. The association of the distance walked in 6 min with preoperative peak oxygen consumption and complications 1 month after colorectal resection. Anaesthesia. 2013;68(8):811-6. doi:10.1111/anae.12329.

10. Johnson KM, Newman KL, Green PK, et al. Incidence and Risk Factors of Postoperative Mortality and Morbidity After Elective Versus Emergent Abdominal Surgery in a National Sample of 8193 Patients With Cirrhosis [published online ahead of print, 2019 Oct 23]. Ann Surg. 2019. doi:10.1097/SLA.0000000000003674.

11. Herdy AH, Ritt LE, Stein R, et al. Cardiopulmonary Exercise Test: Background, Applicability and Interpretation. Arq Bras Cardiol. 2016;107(5):467-81. doi:10.5935/abc.20160171.

12. Older P, Hall A, Hader R. Cardiopulmonary exercise testing as a screening test for perioperative management of major surgery in the elderly. Chest. 1999;116(2):355-62. doi:10.1378/chest.116.2.355.

13. Wilson RJ, Davies S, Yates D, Redman J, Stone M. Impaired functional capacity is associated with all-cause mortality after major elective intra-abdominal surgery. Br J Anaesth. 2010;105(3):297-303. doi:10.1093/bja/aeq128.

14. Lai CW, Minto G, Challand CP, et al. Patients' inability to perform a preoperative cardiopulmonary exercise test or demonstrate an anaerobic threshold is associated with inferior outcomes after major colorectal surgery. Br J Anaesth. 2013;111(4):607-11. doi:10.1093/bja/aet193.

15. Junejo MA, Mason JM, Sheen AJ, et al. Cardiopulmonary exercise testing for preoperative risk assessment before pancreaticoduodenectomy for cancer. Ann Surg Oncol. 2014;21(6):1929-36. doi:10.1245/s10434-014-3493-0.

16. Levett DZH, Jack S, Swart M, et al. Perioperative cardiopulmonary exercise testing (CPET): consensus clinical guidelines on indications, organization, conduct, and physiological interpretation. Br J Anaesth. 2018;120(3):484-500. doi:10.1016/j.bja.2017.10.020.

17. Older $P$, Smith R, Hall A, French C. Preoperative cardiopulmonary risk assessment by cardiopulmonary exercise testing. Crit Care Resusc. 2000;2(3):198-208.

18. World Health Organisation 10th Revision of the International. Statistical Classification of Diseases and Related Health Problems (ICD-10). Released into use in WHO Member States starting in 1994. 
19. Dindo D, Demartines N, Clavien PA. Classification of surgical complications: a new proposal with evaluation in a cohort of 6336 patients and results of a survey. Ann Surg. 2004;240(2):205-13. doi:10.1097/01.sla.0000133083.54934.ae.

20. Grocott MP, Browne JP, Van der Meulen J, et al. The Postoperative Morbidity Survey was validated and used to describe morbidity after major surgery. J Clin Epidemiol. 2007;60(9):919-28. doi:10.1016/j.jclinepi.2006.12.003.

21. Clavien PA, Barkun J, de Oliveira ML, et al. The Clavien-Dindo classification of surgical complications: five-year experience. Ann Surg. 2009;250(2):187-96.

doi:10.1097/SLA.0b013e3181b13ca2.

22. Davies SJ, Francis J, Dilley J, Wilson RJ, Howell SJ, Allgar V. Measuring outcomes after major abdominal surgery during hospitalization: reliability and validity of the Postoperative Morbidity Survey. Perioper Med (Lond). 2013;2(1):1. Published 2013 Feb 4. doi:10.1186/2047-0525-2-1.

23. American Thoracic Society. American College of Chest Physicians. ATS/ACCP Statement on Cardiopulmonary Exercise Testing. Am J Respir Crit Care Med. 2003;167:211-77.

24. Kattan MW, Eastham JA, Stapleton AMF, Wheeler TM, Scardino PT. A preoperative nomogram for disease recurrence following radical prostatectomy for prostate cancer. J Natl Cancer Inst. 1998;90:766-71.

25. Snowden CP, Prentis JM, Anderson HL, et al. Submaximal cardiopulmonary exercise testing predicts complications and hospital length of stay in patients undergoing major elective surgery. Ann Surg. 2010;251(3):535-41. doi:10.1097/SLA.0b013e3181cf811d.

26. American Thoracic Society; American College of Chest Physicians. ATS/ACCP Statement on cardiopulmonary exercise testing [published correction appears in Am J Respir Crit Care Med. 2003 May 15;1451-2]. Am J Respir Crit Care Med. 2003;167(2):211-277. doi:10.1164/rccm.167.2.211.

27. Hennis PJ, Meale PM, Grocott MP. Cardiopulmonary exercise testing for the evaluation of perioperative risk in non-cardiopulmonary surgery. Postgrad Med J. 2011;87(1030):550-7. doi:10.1136/pgmj.2010.107185.

28. Smith TB, Stonell C, Purkayastha S, Paraskevas P. Cardiopulmonary exercise testing as a risk assessment method in non cardio-pulmonary surgery: a systematic review. Anaesthesia. 2009;64(8):883-93. doi:10.1111/j.1365-2044.2009.05983.x.

29. Nagamatsu Y, Sueyoshi S, Tsubuku T, Kawasaki M, Akagi Y. Predicting postoperative exercise capacity after major lung resection. Surg Today. 2015;45(12):1501-8. doi:10.1007/s00595-0151121-7.

30. Brunelli A, Pompili C, Salati M, et al. Preoperative maximum oxygen consumption is associated with prognosis after pulmonary resection in stage I non-small cell lung cancer. Ann Thorac Surg. 2014;98(1):238-42. doi:10.1016/j.athoracsur.2014.04.029.

31. West MA, Lythgoe D, Barben CP, et al. Cardiopulmonary exercise variables are associated with postoperative morbidity after major colonic surgery: a prospective blinded observational study. Br J Anaesth. 2014;112(4):665-71. doi:10.1093/bja/aet408. 
32. Lee L, Schwartzman K, Carli F, et al. The association of the distance walked in 6 min with preoperative peak oxygen consumption and complications 1 month after colorectal resection. Anaesthesia. 2013;68(8):811-6. doi:10.1111/anae.12329.

33. Wilson RJT, Yates DRA, Walkington JP, Davies SJ. Ventilatory inefficiency adversely affects outcomes and longer-term survival after planned colorectal cancer surgery. Br J Anaesth. 2019;123(2):238-45. doi:10.1016/j.bja.2019.01.032.

34. Junejo MA, Mason JM, Sheen AJ, et al. Cardiopulmonary exercise testing for preoperative risk assessment before hepatic resection. Br J Surg. 2012;99(8):1097-104. doi:10.1002/bjs.8773.

35. Kasivisvanathan R, Abbassi-Ghadi N, McLeod AD, et al. Cardiopulmonary exercise testing for predicting postoperative morbidity in patients undergoing hepatic resection surgery. HPB (Oxford). 2015;17(7):637-43. doi:10.1111/hpb.12420.

36. Older PO, Levett DZH. Cardiopulmonary Exercise Testing and Surgery. Ann Am Thorac Soc. 2017;14(Supplement_1):74-83. doi:10.1513/AnnalsATS.201610-780FR.

37. Andrade P, Lopes S. Preoperative Aerobic Capacity - Is There a Role for Routine Evaluation in Liver Transplantation? GE Port J Gastroenterol. 2015;22(3):83-4. doi:10.1016/j.jpge.2015.04.003. Published 2015 May 11.

38. Daabiss M. American Society of Anaesthesiologists physical status classification. Indian J Anaesth. 2011;55(2):111-5. doi:10.4103/0019-5049.79879.

39. Chand M, Armstrong T, Britton G, Nash GF. How and why do we measure surgical risk? J R Soc Med. 2007;100(11):508-12. doi:10.1177/014107680710001113.

40. Stringer WW. Cardiopulmonary exercise testing: current applications. Expert Rev Respir Med. 2010;4(2):179-88. doi:10.1586/ers.10.8.

41. Older PO, Levett DZH. Cardiopulmonary Exercise Testing and Surgery. Ann Am Thorac Soc. 2017;14(Supplement_1):74-83. doi:10.1513/AnnalsATS.201610-780FR.

42. van Diepen S, Youngson E, Ezekowitz JA, McAlister FA. Which risk score best predicts perioperative outcomes in nonvalvular atrial fibrillation patients undergoing noncardiac surgery? Am Heart J. 2014;168(1):60 - 7.e5. doi:10.1016/j.ahj.2014.03.015.

43. Barnett S, Moonesinghe SR. Clinical risk scores to guide perioperative management. Postgrad Med J. 2011;87(1030):535-41. doi:10.1136/pgmj.2010.107169.

44. Stones J, Yates D. Clinical risk assessment tools in anaesthesia. BJA Educ. 2019;19(2):47-53. doi:10.1016/j.bjae.2018.09.009.

45. Wong DJN, Oliver CM, Moonesinghe SR. Predicting postoperative morbidity in adult elective surgical patients using the Surgical Outcome Risk Tool (SORT). Br J Anaesth. 2017;119(1):95-105. doi:10.1093/bja/aex117.

46. Tjeertes EK, Hoeks SE, Beks SB, Valentijn TM, Hoofwijk AG, Stolker RJ. Obesity-a risk factor for postoperative complications in general surgery? [published correction appears in BMC Anesthesiol. 2015;15:155. Tjeertes, Elke E K M [corrected to Tjeertes, E K M]; Hoeks, Sanne S E [corrected to Hoeks, S E]; Beks, Sabine S B J C [corrected to Beks, S B J]; Valentign, Tabita T M [corrected to Valentijn, T 
M]; Hoofwijk, Anton A G M [corrected to Hoofwijk, A G M]; Sto]. BMC Anesthesiol. 2015;15:112. Published 2015 Jul 31. doi:10.1186/s12871-015-0096-7.

47. Sugie M, Harada K, Takahashi T, et al. Relationship between skeletal muscle mass and cardiac function during exercise in community-dwelling older adults. ESC Heart Fail. 2017;4(4):409-16. doi:10.1002/ehf2.12158.

48. Kim CH, Wheatley CM, Behnia M, Johnson BD. The Effect of Aging on Relationships between Lean Body Mass and VO2max in Rowers. PLoS One. 2016;11(8):e0160275. doi:10.1371/journal.pone.0160275. Published 2016 Aug 1.

49. Buia A, Stockhausen F, Hanisch E. Laparoscopic surgery: A qualified systematic review. World J Methodol. 2015;5(4):238-54. doi:10.5662/wjm.v5.i4.238. Published 2015 Dec 26.

50. Wang S, Shi N, You L, Dai M, Zhao Y. Minimally invasive surgical approach versus open procedure for pancreaticoduodenectomy: A systematic review and meta-analysis. Medicine. 2017;96(50):e8619. doi:10.1097/MD.0000000000008619.

51. Kavalukas SL, Ghuman A, Sharp SP, Wexner SD. Robotic or laparoscopic surgery for rectal cancer which is the best answer? a comprehensive review of non-oncological outcomes and learning curve. Mini-invasive Surg. 2020;4:61. doi:10.20517/2574-1225.2020.71.

52. Ashrafian $\mathrm{H}$, Clancy $\mathrm{O}$, Grover V, Darzi A. The evolution of robotic surgery: surgical and anaesthetic aspects. Br J Anaesth. 2017;119(suppl_1):i72-84. doi:10.1093/bja/aex383.

53. Otto JM, Levett DZH, Grocott MPW. Cardiopulmonary Exercise Testing for Preoperative Evaluation: What Does the Future Hold? Curr Anesthesiol Rep. 2020;10:1-11. doi.org/10.1007/s40140-02000373-x.

54. American Thoracic Society; American College of Chest Physicians. ATS/ACCP Statement on cardiopulmonary exercise testing [published correction appears in Am J Respir Crit Care Med. 2003 May 15;1451-2]. Am J Respir Crit Care Med. 2003;167(2):211-277. doi:10.1164/rccm.167.2.211.

55. Beltz NM, Gibson AL, Janot JM, Kravitz L, Mermier CM, Dalleck LC. Graded Exercise Testing Protocols for the Determination of $\mathrm{VO}_{2}$ max: Historical Perspectives, Progress, and Future Considerations. J Sports Med (Hindawi Publ Corp). 2016;2016:3968393. doi:10.1155/2016/3968393.

56. Jaffer AK, Grant PJ. Perioperative Medicine: Medical Consultation and Co-Management. WileyBlackwell; 2012.

57. Hawkins MN, Raven PB, Snell PG, Stray-Gundersen J, Levine BD. Maximal oxygen uptake as a parametric measure of cardiorespiratory capacity [published correction appears in Med Sci Sports Exerc. 2007 Mar;39(3):574]. Med Sci Sports Exerc. 2007;39(1):103-107. doi:10.1249/01.mss.0000241641.75101.64.

58. Salati M, Brunelli A. Risk Stratification in Lung Resection. Curr Surg Rep. 2016;4(11):37. doi:10.1007/s40137-016-0158-x.

\section{Appendix}


Cardiopulmonary Exercise Test (CPET) is being increasingly used to evaluate patients' functional capacity pre-operatively. ${ }^{53} \mathrm{It}$ is one of the few non-invasive tests available that can yield a range of reproducible physiological data reflective of physical fitness. ${ }^{54}$ With a graded exercise mechanism that assess physical and psychological wellbeing, it provides a measurement of maximal exercise capacity as well as identification of the causes that limit exercise capacity. ${ }^{55}$ CPET has been shown to be a valuable tool in perioperative morbidity and mortality risk prediction through estimation of pre-operative aerobic fitness level. Multiple studies have shown that patients with diminished cardiopulmonary reserve are more predisposed to peri-operative complications, resulting in short and/or long-term patient suffering while adding significant financial costs to the healthcare system. ${ }^{56}$ This problem is exacerbated with an ageing population presenting with more complexity. As a result self-paced, graded exercise testing provide an individualised assessment of physical fitness.

Maximal Oxygen Consumption at Maximal Exercise Capacity (VO2 Max) is the highest VO2 obtained during CPET testing and reflects exercise capacity as a percentage of the predicted value for maximum oxygen consumption. Introduced in 1923, Hill and Lupton defined VO2 Max as "the oxygen intake during an exercise intensity at which actual oxygen intake reaches a maximum beyond which no increase in effort can raise it." It is the point at which VO2 does not continue to increase, or only increase by a trivial amount, despite further increases in work rate. ${ }^{57}$ At this stage of CPET testing, the VO2 reaches a plateau phase when VO2 is plotted as a function of work rate. Ventilatory Equivalent for Carbon Dioxide at Anaerobic Threshold (AT VE/VCO2) is the ventilatory equivalent for carbon dioxide defined as the ratio of minute ventilation to carbon dioxide production reported at AT. In a review by Salati et al. published in 2016, recent findings suggested that patients with $\mathrm{VO} 2 \mathrm{Max}>20 \mathrm{ml} / \mathrm{kg} / \mathrm{min}$ were regarded at low risk, while those with VO2 Max $<10 \mathrm{ml} / \mathrm{kg} / \mathrm{min}$ were deemed at high risk. Where patients had a VO2 Max between 10 and $20 \mathrm{~mL} / \mathrm{kg} / \mathrm{min}$, further risk stratification by the VE/VCO2 slope was indicated. VE/VCO2 further categorised patients into intermediate-low risk with values $<35 \mathrm{ml} / \mathrm{kg} / \mathrm{min}$, while values above 35 $\mathrm{ml} / \mathrm{kg} / \mathrm{min}$ indicates an intermediate-high risk. ${ }^{58}$

\section{Figures}




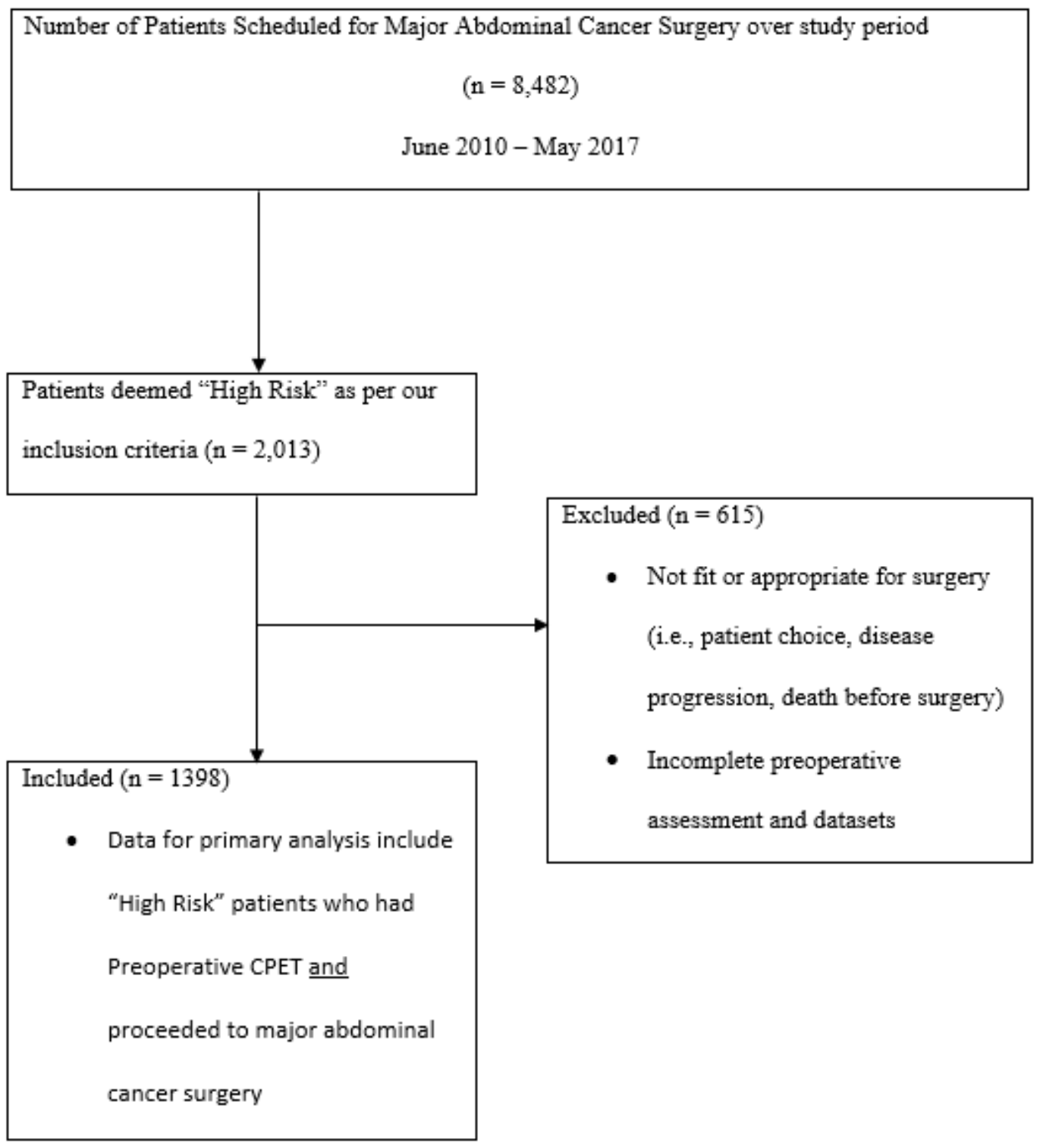

\section{Figure 1}

Modified CONSORT flow diagram for patients in the primary analysis. CPET, cardiopulmonary exercise testing 


\section{VARIABLES}

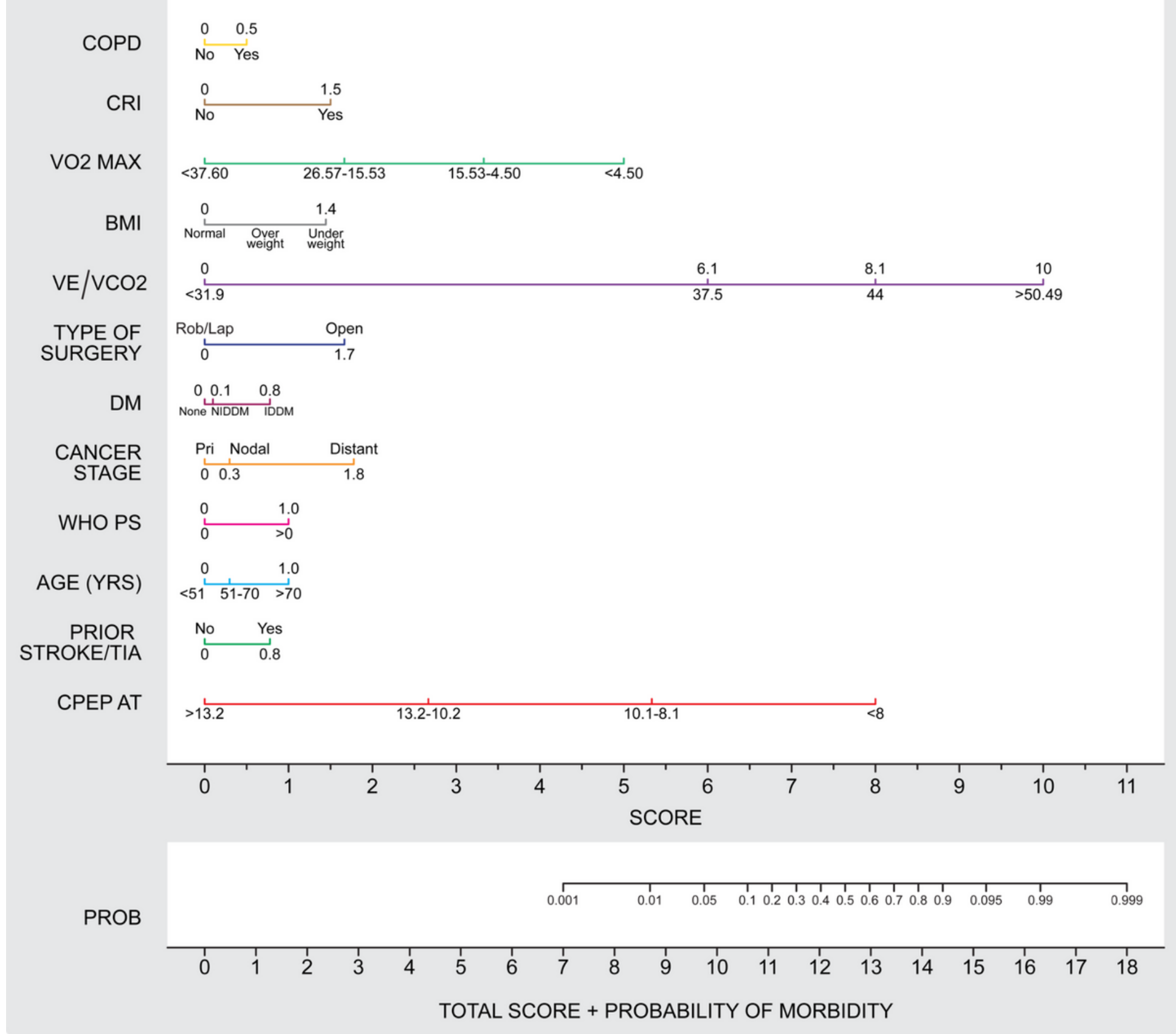

\section{Figure 2}

Fitted model variables - NOMOGRAPH Fitted model variables - Nomograph. COPD, Chronic Obstructive Pulmonary Disease. CRI, Chronic Renal Impairment. VO2 Max, Maximal Oxygen Consumption at Maximal Exercise Capacity. BMI, Body Mass Index. VE/VCO2 - Ventilatory Equivalent for Carbon Dioxide; VE/VCO2 values in this diagram refers to VE/VCO2 taken at Anaerobic Threshold (AT VE/VCO2). Rob/Lap, Roboticassisted or laparoscopic-assisted surgery. DM, Diabetes Mellitus. NIDDM, Non-Insulin Dependent DM. IDDM, Insulin Dependent DM. Pri, Primary. WHO PS, World Health Organisation Point Score. TIA Transient Ischaemic Attack. CPET, Cardiopulmonary Exercise Testing. AT, Anaerobic Threshold 